\title{
How to design compelling Virtual Reality or Augmented Reality experience?
}

\author{
Simon Richir ${ }^{1}$, Philippe Fuchs ${ }^{2}$, Domitile Lourdeaux ${ }^{3}$, \\ Dominique Millet $^{4}$, Cédric Buche ${ }^{5}$, Ronan Querrec ${ }^{5}$ \\ ${ }^{1}$ Arts et Metiers ParisTech, LAMPA, 2 Bd du Ronceray, 49000 Angers - France \\ ${ }^{2}$ Mines ParisTech, VR\&RA Team \\ ${ }^{3}$ UTC, Heudiasyc-UMR6599 \\ ${ }^{4}$ Supméca Toulon, 83000 Toulon \\ ${ }^{5}$ ENIB, LAB-STICC-UMR3192 \\ Contact author: simon.richir@ensam.eu
}

\begin{abstract}
The convergence of technologies currently observed in the field of Virtual Reality, Augmented Reality, robotics and consumer electronic reinforces the trend of new applications appearing every day. But when transferring knowledge acquired from research to businesses, research laboratories are often at a loss because of a lack of knowledge of the design and integration processes in creating an industrial scale product. In fact, the innovation approaches that take a good idea from the laboratory to a successful industrial product are often little known to researchers.
\end{abstract}

The objective of this paper is to present the results of the work of several research teams that have finalized a working method for researchers and manufacturers that allow them to design virtual or augmented reality systems and enable their users to enjoy "a compelling Virtual Reality experience". That approach, called "the I2I method", present 11 phases from "Establishing technological and competitive intelligence and industrial property" to "Improvements" through the "Definition of the Behavioral Interface, Virtual Environment and Behavioral Software Assistance". As a result of the experience gained by various research teams, this design approach benefits from contributions from current Virtual Reality \& Augmented Reality research. Our objective is to validate and continuously move such multidisciplinary design team methods forward.

Index Terms - Virtual reality, design process, human centered design, methodology, innovation, immersion, interaction

\section{INTRODUCTION}

The video games sector, where production costs and turnover now exceed those of the cinema industry, use some structured, formalised approach to developing new products that all companies in the sector have taken on board. This is not yet the case with regard to producing systems making use of virtual reality technologies (VR). The I2I method (Immersion \& Interaction for Innovation), finalised by two ParisTech research teams, aims to guide designers of such systems to allow them to create a product that best responds to the needs of their future users, while respecting the best balance of costs, timescales, quality and innovation [1].

We offer a method that makes it possible to design an innovative system, with optimal cost, timescales and quality, in which the user finds an useful simulation of certain aspects of the real world. Those systems apply to training, simulation, industry, architecture or health fields. In this sort of system, the user makes use of all his/her senses, intuitively understanding what to do. The disciplines involved in meeting the challenge are numerous and not limited to the usual design or virtual reality jobs [2]. Other sciences have to be understood and incorporated so that a (real) user carries out the tasks virtually, and is able to extract the information from it that can be extrapolated into a real environment. Involving disciplines such as ergonomics, cognition or psychology then becomes necessary. Here we are tackling a scientific obstacle that touches on the interdisciplinary complexity of implementing scientific and virtual reality techniques (VR).

\section{DESIGNING VIRTUAL REALITY SYSTEMS}

We noted the present lack of formal methods for designing systems integrating Virtual Reality. During the "Virtual Realities", Dagstuhl Seminar 08231 in June 2008, the topic "Experience Design" was considered a largely important one in the Virtual Reality field [3] [4] [5].

The bibliography on the subject is mainly made up of descriptions of the experimental development of 
systems or evaluations of systems marketed by a few companies [6] [7]. These companies do not, however, reveal the methods they used to get to the point of marketing their products, either out of a concern for confidentiality, or because quite simply they had not formalised them. A look at the winners of the "Laval Virtual Awards" over the last 17 years, which each year reward the best world achievements in the field of virtual reality, demonstrates that the majority of the applications are rarely developed and finalised on the basis of standardised processes.

However, a distinction should be made between the methods used to design virtual reality systems as a whole, which is the subject of our article, and methods for interfacing virtual reality systems (3D User Interface design) which have been subject to numerous scientific studies: in particular the work of Chen \& Bowman [8].

Fuchs and his colleagues [9] firstly developed an initial method with the objective of effectively interfacing the user in a virtual environment in line with the desired activities. This " $3 \mathrm{I}^{2}$ " method is central to the general design method but is limited to realising the desired activities. It is based on a three level analysis of user immersion and interaction $\left(\mathrm{I}^{2}\right)$, hence its name $3 \mathrm{I}^{2}$ (or the " $3 \mathrm{I}^{2}$ " model): sensorimotor immersion and interaction $\left(\mathrm{I}^{2}\right)$, cognitive $\mathrm{I}^{2}$ and functional $\mathrm{I}^{2}$. This method gives us a nucleus on which to base a general design method for applications employing virtual reality techniques (VR or RV -"Réalité Virtuelle" in French) - a method based on an innovation approach. Before presenting this general method in detail, the following paragraphs outline the main concepts in the $3 \mathrm{I}^{2}$ interfacing method.

\section{ESSENTIAL CONCEPTS IN THE 3I'2 INTERFACING METHOD}

\section{a. 3.1 Behavioural interfacing}

In any system using Virtual Reality, the user is fundamentally at the centre of the mechanism as the virtual application is aimed at the user. In establishing

\footnotetext{
${ }^{1}$ Simon Richir is the co-founder and scientific director of Laval Virtual. This event every year since 1999 has had 10,000 to 15,000 visitors. Simon Richir also manages the organisation of VRIC, Virtual Reality International Conference, of student competitions and of the Laval Virtual Awards. www.laval-virtual.org
}

the virtual environment and its interfaces (Figure 1) it is therefore necessary to use a human centred approach (focusing on the person) starting with the loop "perception, cognition, action", rather than a technical centred approach (focusing on the computer) [10].

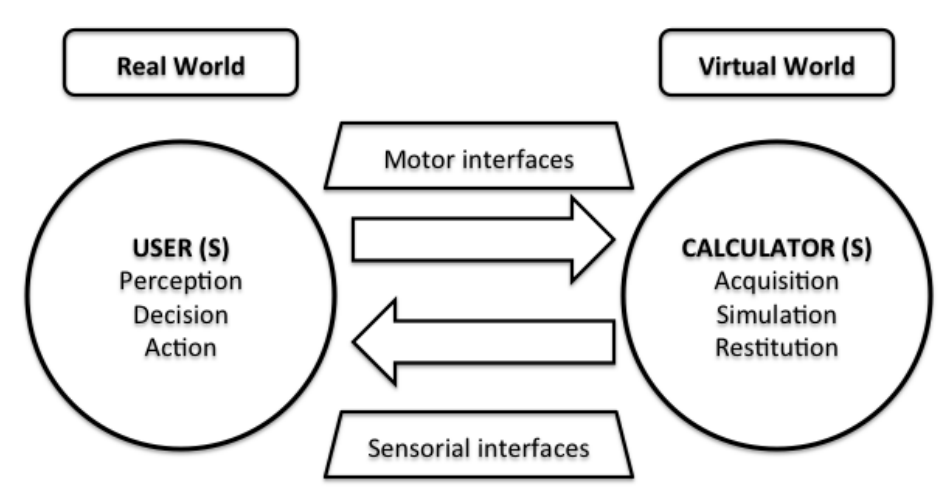

Figure 1. The "perception, cognition, action" loop passing by the virtual mode

We will use the term Behavioural Interfaces (BI) rather than physical interfaces or man machine interfaces in order to clearly differentiate them. For the purposes of our definition, the behavioural interface is a mechanism that partly exploits the user's perception and / or natural motor skills, which are the basis of human behaviour. At a theoretical level, the "behavourial interfaces" are designed to transmit sensorial stimuli from the computer to the person and vice versa, the "motor interfaces" are designed to transmit the motor responses from man to the computer. Some sensorimotor interfaces, force feedback interfaces, transmit the motor responses and, as a reaction, sensorial stimuli are fed back by the computer (Figure 2). 


\section{Behavourial Interfaces}

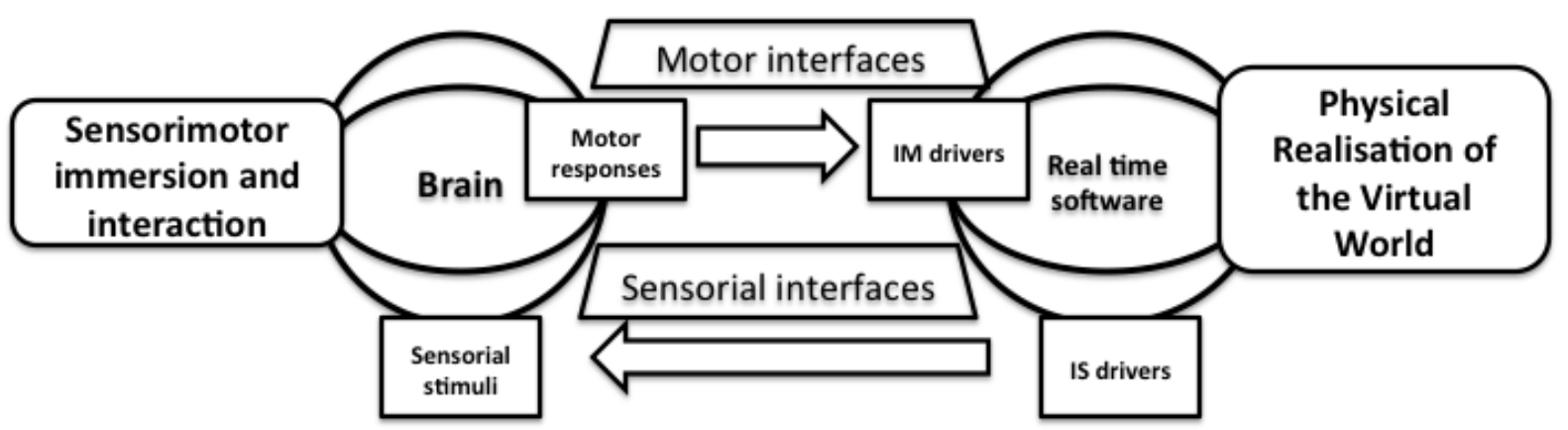

Figure 2. Technical centred schema of sensorimotor immersion and interaction

Behavioural interfaces pose a similar interfacing problem to that of an operator with a machine or tool. In this case it is not just a question of thinking through the interface at a physical level (control buttons and information feedback on actions), but also of understanding which mental thought and action models the person will use. As Rabardel described in his instrumental approach to interfacing [11], the instrument (the interface) is a mediator for activity.

The user must have an activity (a behaviour) in the virtual world. The interface must be transparent to him or her. In concrete terms, this activity is physically realised by means of the user's motor skills and an effective perception between the person and the behavioural interfaces. Physically, these rely on artefacts (or instruments) and are used depending on the user's mental schemas.

To facilitate the use of behavioural interfaces we should not lose sight of the fact that in the case of systems incorporating Virtual Reality techniques, the designer can help the user to employ these interfaces efficiently. There are many possibilities that can be exploited and programmed. For example, specific restriction to the movement of objects can be added to help the user to obtain the desired motor skills (the bearing surface of an object close to a table automatically positions itself parallel to the table when it approaches the surface) [12]. All these aids to using interfaces are gathered together under the term "Behavioural Software Assistance" (BSA). Consequently, the coupling between the interface and the virtual world has to be designed and programmed.
The sensorial perception imposed to the user should be natural, but it has the disadvantage of being nearly always partial and more or less coherent. For example, the perception of movement uses vision, sensitivity to force, the inner ear (semicircular canals, otoliths) and touch. Depending on the behavioural interface chosen, certain types of sensorial receptors will not be stimulated, others will be but with characteristics different from those that can be detected by human beings in a real environment. All the effective stimulations could lead to inconsistencies that the brain has to manage or it will fail. The problem is similar for effective motor skills. Difficulties caused by inconsistencies are inherent to virtual reality techniques and in many cases will always be complicated to resolve. Can the brain adapt or will it refuse? Experiments conducted by cognitive science specialists and their knowledge may help us to either confirm or reject our designs.

There are several ways of making a subject cognitively immerge and (inter)act in a virtual environment as Imported Behavioural Schema (IBS) or Metaphors.

\section{b. Imported Behavioural Schema}

The first idea that comes to mind is to offer the user relatively natural interaction and immersion. In this case, we talk about pseudo natural immersion. What does this concept cover? Let us take an example: in a virtual store, the consumer wishes to move through the aisles. We offer a real trolley as a behavioural interface that will be pushed and directed in front of a screen (in a real limited space). In this case, the consumer will unconsciously and naturally exploit one of the automatic reactions already acquired during shopping in a real store in the virtual store with a 
few sensorimotor differences related to the fact that the displacement of the trolley is not real (Figure 3). Hence the prefix "pseudo" employed for this type of immersion and interaction.

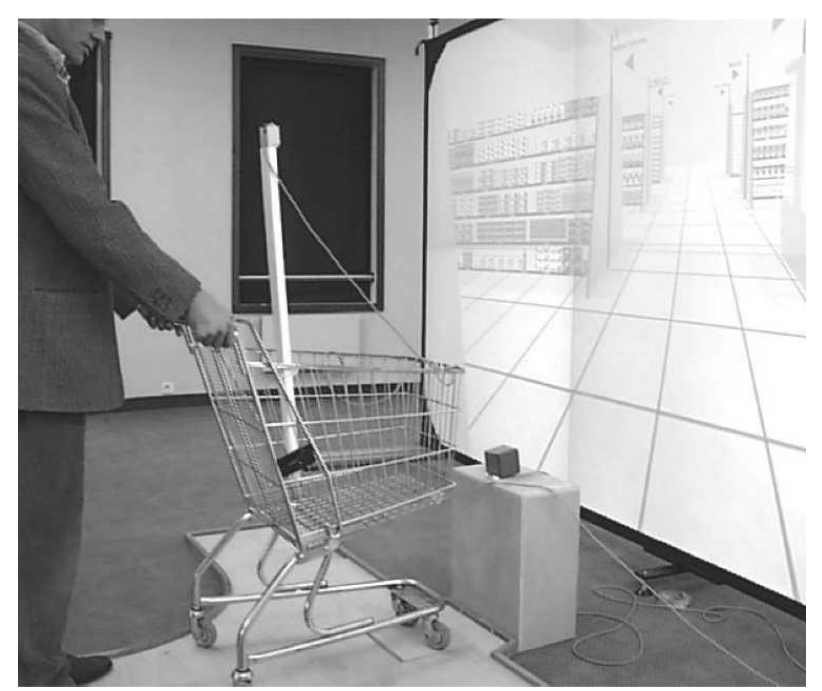

Figure 3. Behavioural interface using a trolley fitted with instruments for moving in a virtual store (photo Mines ParisTech)

To clarify these cognitive immersions and interactions $\left(\mathrm{I}^{2}\right)$, the user will make use of a sensorimotor schema that has been assimilated in the real world. The notion of a schema was put forward by the psychologist Piaget [13]. He considered that in analysing the birth of intelligence in a child, in particular the sensorimotor aspect, schemas are resources the subject uses to help assimilate any situations and objects with which $\mathrm{s} / \mathrm{he}$ is confronted. For Piaget, a schema is a structured group of action characteristics that can be generalised and which makes it possible to repeat the action or to apply it to new content (as the consumer does manipulating the trolley in conditions close to those encountered in the real world). A schema is therefore the mental organisation of actions as they can be transferred or generalised when the action is repeated in similar circumstances. They correspond to the stable aspects in various classes of situations. Use schemas have a personal dimension proper to each individual; they are part of the subject's personal memory in the form of resources that can be mobilised. They also have a social dimension: they are common to all or many members in a particular social group, an organisation, a working environment. Consequently is appropriate to also consider them as social use schemas, such as resources inscribed in the memory of groupings [11]. We base our approach on this concept with a view to obtaining the behavioural interfaces, offering pseudo natural immersion and interaction. The behavioural interface is in this case a mixed entity comprising both an artefact (the physical mechanism) and a schema that we call "Imported
Behavioural Schema" (IBS). The schema is imported from the real environment and is transposed and adapted into a virtual environment.

As Piaget [13] described:

- A schema can be reproduced: it contains the conditions for recognising the situations in which it is applicable;

- A schema is assimilating: it can be applied in new situations;

- A schema has a purpose;

- A schema is not declarative knowledge (it is used and assimilated unconsciously).

To clarify these notions, let us take the example of handling an object. The associated schema is not the particular process of the movements and perceptions. The general framework makes it possible to reproduce the schema in different circumstances and produce a variety of different results. The arm is extended or the hand opened to different extents, depending on how far away the object is and its size. Whatever the object may be, it is always the same schema used to handle it. Here we just vary the sensorimotor parameters for the same schema.

The Imported Behavioural Schema (IBS) cannot be isolated. It has an interdependent relationship with the artefact (physical interface either developed or bought) which, in a given application, is associated with it. But the same schema may be exploited with different artefacts and vice versa: for handling in a 3D space, a "tracker" (position sensor) with six degrees of freedom or a data glove can be used with the same schema. An imported behavioural schema, used in a virtual environment is often distorted in relation to the real environment schema. The sensorimotor functions used may in part be different from those in the real world. And not only the sensorimotor parameters vary. For example, for grasping and handling objects in the virtual store, the sensorimotor functions are modified: with a "tracker" with 6 degrees of freedom, the user can virtually grasp the object and handle it. But we are looking at the screen and not at our hands and the object is grasped without us really closing our hands. However, if there is any distortion it does not stop the user unconsciously making use of the same schema.

\section{c. Metaphor with or without sensorimotor substitution}

Another possibility is to employ a metaphor (symbolic image) instead of making use of the user's acquired Imported Behavioural Schema (IBS). We can offer the subject, usually visually, a symbolic image of the desired action or perception 
(Figure 4). For example in a virtual store, we can suggest the consumer simply clicks on the store plan image to indicate where s/he wants to move in the store. The action becomes symbolic and is no longer representative of the sensorimotor action in a real store, the immersion and the interaction is less natural.

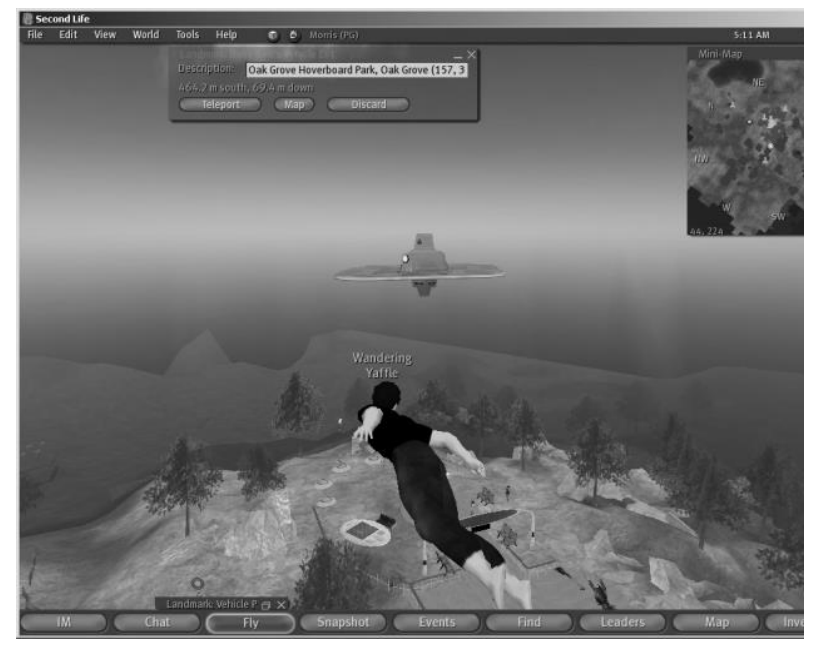

Figure 4. Using the metaphor of a map for rapid navigation in $2^{\text {nd }}$ Life

If there are difficulties using an IBS or a metaphor employing the same senses and motor responses as in the real world, another possibility is to use a metaphor using sensorial substitution or a metaphor using motor substitution. For example, it is often difficult to transmit the effort to the user with a force feedback interface. The difficulty can be circumvented by substituting the effort perception sense by using hearing: a sound is made when the object user is handling encounters an obstacle. This sensorial substitution is effective in the present case if it is a question of just warning the user about an obstacle. However, if the perception of the intensity and direction of effort is important for the application (assembling parts for example), the substitution is not appropriate. Substitution may also use motor skills: instead of moving by walking physically, the user can order virtual movement by voice ("I want to go forwards, backwards, turn left, turn right", etc) with a voice command interface and with a dynamic view of the virtual world.

The use of metaphors may require greater cognitive effort if the symbolic metaphor is not known to the users. They have to make an effort to understand and assimilate it, so that through use it becomes a use schema. But an Imported Behavioural Schema (IBS) may also require a certain effort as it has to be adapted to a virtual world with a particular artefact and with the restrictions of sensorimotor inconsistency. We will use a metaphor instead of an IBS if there are theoretical, economic or technical issues. In practice, we can sometimes associate metaphors and IBS depending on the type and importance of the desired activities.

Metaphors with sensorial or motor substitution distance the virtual action from the real action.

\section{d. Use of schemas, metaphors or sensorimotor substitutions}

It could be thought at first sight that it is preferable to exploit an Imported Behavioural Schema (IBS) instead of a "metaphor". But above all the choice depends on the objectives of the application (which will be specified at the functional immersion and interaction level, $\mathrm{I}^{2} \mathrm{f}$, see below) and on the users capacities.

Instead of exploiting a user's learned sensorimotor behaviour, we can offer, in general visually, a symbolic vision of the desired action or of the perception. Using metaphors may demand more cognitive effort if the symbolic metaphor is unknown to the users. They have to make the effort to understand and assimilate it so that through use it becomes the use schema. However, an Imported Behavioural Schema (IBS) may also require a certain effort as it has to be adapted to a virtual world with a particular artefact and with the restrictions of sensorimotor inconsistency. We will use a metaphor instead of an IBS if there are theoretical, economic or technical issues. In practice, we can sometimes associate metaphors and IBS depending the type and importance of the desired activities.

Metaphors with sensorial or motor substitution distance the virtual action from the real action. However, immersion and interaction will not automatically be more difficult and will take longer to learn, because the virtual action is less similar to the real action. If the consequence of employing an IBS and not a metaphor, with or without substitution, is an interface that is overly technically imperfect, the action will be difficult. This risks putting the user's action at a greater disadvantage, even if it is closer to the similar action in the real world.

Other criteria are important in deciding whether or not to exploit an Imported Behavioural Schema (IBS). In the case where the sensorimotor action is itself being studied, which is often encountered in product design (where a virtual product is being evaluated as if it were real), using an IBS in the virtual world is essential and should "physically" come closer to the schema in the real environment: for example, manipulating the control buttons on an instrument panel to make an ergonomic study of the controls. However, if the sensorimotor action only serves to indicate what the subject wants, the use of an IBS is not essential and can be compared with a metaphor, with or without sensorial or motor 
substitution. The application therefore has to be analysed in order to find out whether it is a sensorimotor level activity (schema required) or a simple indication of activity.

Another example is that of simulating the process of assembling (or dismantling) parts in order to analyse the assembly or maintenance of a mechanism. The objective of the simulation is not identical, for example, for the following three different target scenarios:

- Verifying that the dimensions of an object allow the mechanism to be assembled in spite of the size of the parts (establishing a possible trajectory for the object between the other parts);

- Looking at the possibility of manual assembly by an operator (establishing a possible trajectory for the object and the hands of the operator between the other parts);

- Ergonomically analysing the assembly of the mechanism by an operator (establishing a suitable position for the operator, time and effort required for assembly).

In the first case, the object just has to be moved with six degrees of freedom - it does not much matter how - in order to apply orders to move the object in the Virtual Reality system. In the third case, the ergonomic study in principle imposes the use of force feedback interfaces and an IBS.

Although the above mentioned examples have been presented in order to differentiate the choices to be made between using an Imported Behavioural Schema (IBS) or a metaphor, with or without substitution, there are applications where the choice between the different possibilities is not exclusive. We could therefore consider that there is a continuum of solutions ranging between extreme cases (exclusively IBS to purely metaphor) for the cognitive interaction and immersion processes. The use of Imported Behavioural Schemas or metaphors, with or without sensorial or motor substitution, is a debate that is far from being over in the scientific community.

After having presented the psychocognitive approach and the interdisciplinary technological / cognitive approach in systems incorporating Virtual Reality, we are going back to the world of designers who we are trying to offer a reliable method that guarantees a system design that properly takes account of all the technical and use related aspects.

\section{THE I2I METHOD \\ e. Three levels of immersion and interaction}

The software and hardware production of a system integrating virtual reality cannot be designed without analyzing human behavior in a virtual world. From that point on, it becomes absolutely indispensible to work with cognitive science experts. Their role is to establish:

- The psychophysical characteristics of the user's senses and the motor responses;

- The conceptual and pragmatic differences between the schemas and metaphors employed in the system;

- The Behavioral Software Assistance needed to help the system user.

The limits of human behavior in relation to the solutions chosen by the system designers, in particular the human capacity to resolve any sensorimotor inconsistencies [14].

Our I'I approach is built around analyzing and modeling the subject's immersion and interaction in the virtual environment (Figure 5), developed at the Ecole des Mines ParisTech [15]. Modeling the subject's immersion and interaction should be examined on three levels:

- At a physical level, we talk about sensorimotor immersion and interaction ( $\left.\mathrm{I}^{2} \mathrm{sm}\right)$, as physically the computer is connected to the user through the user's senses and motor responses via the UPI (User Perceptual Interface) [5]. This level of immersion and interaction is quantifiable in relation to the characteristics of the senses and the motor responses being exploited.

- The user must be mentally immersed in the virtual world. The "lower" level of sensorimotor immersion and interaction facing the user should be mentally invisible (transparent). In this case we talk about cognitive immersion and interaction $\left(\mathrm{I}^{2} \mathrm{c}\right)$. The cognitive processes (schemas, metaphors, substitutions) related to the interface are situated at this level.

- At a third level relating to the application of virtual reality, the objective is to endeavor to produce the immersion of the user for a given task (or a functionality). Here we talk about functional immersion and interaction ( $\left.\mathrm{I}^{2} \mathrm{f}\right)$.

This breakdown allows us to more effectively clarify the various issues encountered during the subject's (human being) immersion and interaction. They are closely linked and are not in conflict. The basis of our approach is this three level hierarchical model as well as a horizontal breakdown between the subject and the virtual world: in parallel to the 
person's various $\mathrm{I}^{2} \mathrm{sm}$ and $\mathrm{I}^{2} \mathrm{c}$ levels, we have two levels of software functioning for the virtual world. Symmetrically with the $\mathrm{I}^{2} \mathrm{sm}$, the computer has to manage the real time software part, allowing the physical production of the virtual world. This concerns the simulation based on physical laws (mechanical, optical, biomechanical, etc.) acting on the objects and animated beings.

Given the $I^{2} \mathrm{c}$, the software part has to manage the behavior modeling for the virtual world. This software part has to provide a simulation of the behavior of the animated beings and the Behavioral Software Assistance (BSA) associated with the schemas used, in order to facilitate the person's cognitive $\mathrm{I}^{2} \mathrm{c}$.

In relation to the application and its objectives and at the $I^{2} \mathrm{f}$ level, the following question must be asked: what must the user do? The subject's activities can always be broken down into a few basic behaviors we call "Virtual Behavioral Primitives" (VBP). Therefore at the $\mathrm{I}^{2} \mathrm{f}$ level the VBP and their specificities must be properly established. Whatever the application, they can be grouped into four categories:

- Observe the virtual world;

- Move around in the virtual world;

- Interact in the virtual world;

- Communicate with others or with the application.

Our analysis of the modeling of the subject's immersion and interaction in the virtual environment is summed up by following schema

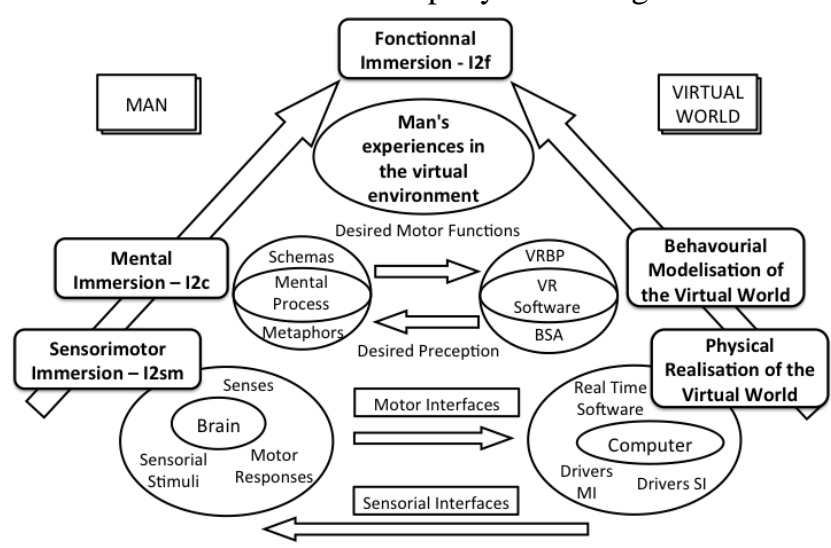

(Figure 5).

Figure 5. Immersion and interaction of man in a virtual environment [15].

\section{f. 1.2 The various phases of the I2I method}

After having described the theoretical foundations of our method, we are now going to describe its 11 phases in detail (Figure 6). In order to facilitate understanding of the method, we will imagine we are in the following situation: a project manager has just been told by his company to put a team and design a process to create, develop and evaluate a system using virtual reality technologies.

We will also present examples of concrete applications in order to facilitate understanding of our method, in particular a virtual store application aimed at studying consumer behavior.

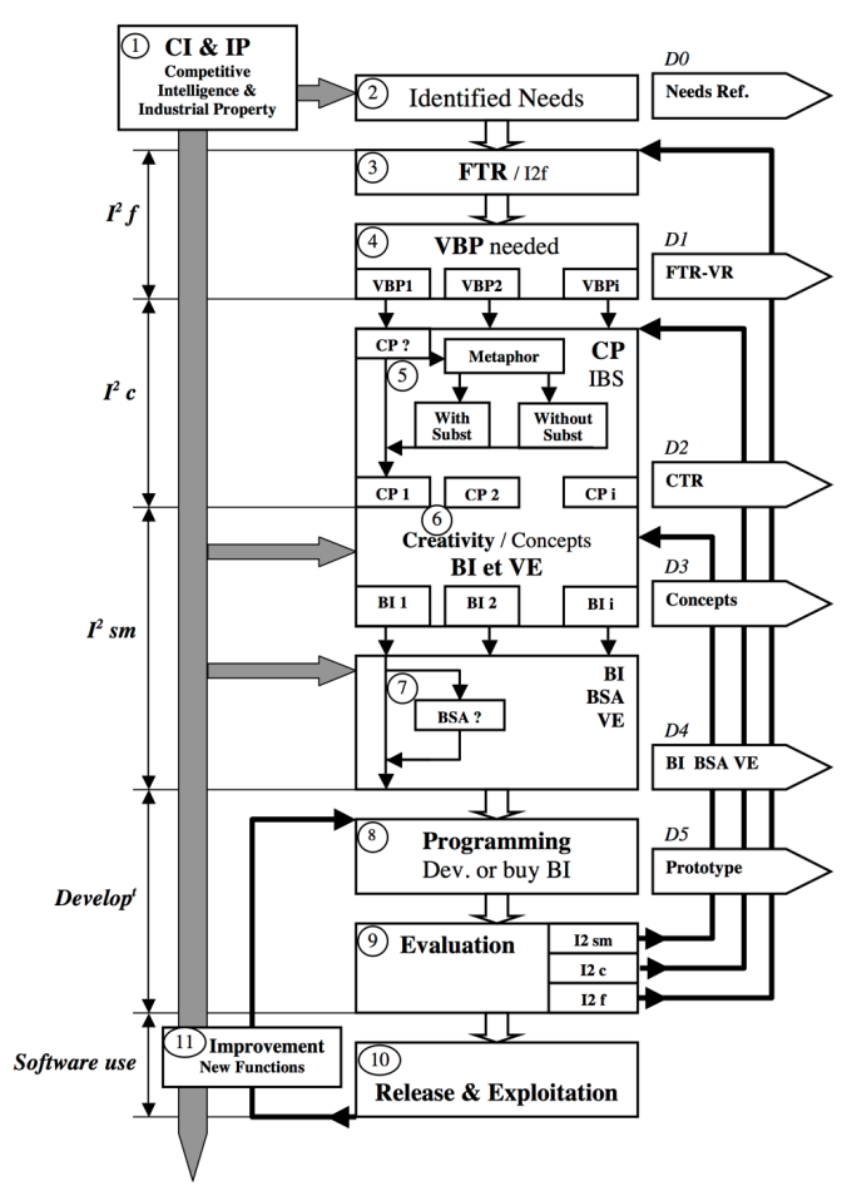

Figure 6 . The I'I method - a virtual reality system design method [2]

$\boldsymbol{B I}=$ Behavioral Interface $(I C) ; \boldsymbol{B S A}=$ Behavioral

Software Assistance $(A L C) ; \boldsymbol{I B S}=$ Imported

Behavioral Schema $($ SCI $) ; \boldsymbol{F T R}=$ Functional

Terms of Reference $(C D C F) ; \boldsymbol{B I}=$ Behavioral

Interfaces $(I C) ; \boldsymbol{V E}=$ Virtual Environment $; \boldsymbol{V B P}$

$=$ Virtual Behavioral Primitives $(P C V) ; \boldsymbol{C P}=$

Cognitive Processes $(P C) ; \boldsymbol{C T R}=$ Cognitive

Terms of Reference $; \boldsymbol{U P I}=$ User Perceptual

Interface $; \boldsymbol{I}^{2} \boldsymbol{f}=$ Functional Immersion \&

Interaction $; \boldsymbol{I}^{2} \boldsymbol{c}=$ Cognitive Immersion $\&$

Interaction $; \boldsymbol{I} \boldsymbol{f}=$ Sensorimotor Immersion \& Interaction. 


\section{PHASE 1: Establishing technological and competitive intelligence and industrial property.}

We can never insist enough on the importance of technological intelligence while developing systems incorporating new technologies [16]. Setting up technological and competitive intelligence systems can make use of an existing service in the company, which we will ask to monitor a certain number of areas and companies. It can also be subcontracted to an outside organization or entrusted to one or several members of the project team. This initial phase is important and furthermore continues throughout the life of the project. It may also provide an opportunity for the team to update its knowledge before starting the project. The team will therefore discover the existence of systems corresponding to the project goal or may even render the project obsolete.

\section{PHASE 2: Identifying and validating the need to design the system}

Obvious for any designer, this preliminary phase to launching the project is aimed at properly defining the sponsor's needs in relation to developing the system: why is it needed? What is the system supposed to do that is new? What actions, which cannot be done in the real world, does the system make possible? What does the system make it possible to improve? What are the benefits for the company? What are the key factors for success? (= How will the success of the project be judged?) Is the sponsor aware of the cost of developing such a system? What will the return on investment be in relation to a real system? Is there an existing system that is sufficient? Have any other similar systems already been developed? What will be the uses in the future? Do we have access to sufficient human, technical and financial resources? [17]

Virtual reality imposes specific requirements that will need to be satisfied in order to determine whether it will be able to meet the sponsor's needs. In the first instance we should point out that the objective of virtual reality is not necessarily to simulate reality as best possible. It is however an idea that is often generally accepted [18]. The purpose of virtual reality could be to symbolically represent phenomena, concepts or simulate certain aspects of reality. In a very general way, we can state that virtual reality capability range make it possible to change time (past or future), place (geographically distant or virtual collaborative space) or type of interaction (symbolic or natural) [9].

This phase ends by constituting the design team around a project leader with all the competencies required to achieve the goals of the project.
PHASE 3: Functional Terms of Reference (FTR) and establishing the functional immersions and interactions (I2f).

We will not be expanding here on the method for drawing up a Functional Terms of Reference (FTR). Those interested should refer to the numerous publications and course materials on the subject [19]. The FTR is produced by the design team and finalized by the project leader. It aims to properly formalize ("translate") the design need for the new system and in functional terms clearly define the primary and secondary functions (or "compulsory functions") that the future system should provide. In the framework of developing systems incorporating Virtual Reality, this phase makes it possible to define the I2f (functional immersions and interactions), as recommended in the "3I2" method [9]. For example, a virtual store must "allow the person to choose a product from among others on a shelf and be able to freely observe him or her" (Figure 7). This primary function ("allow...") is detailed and quantified in the Functional Terms of Reference (FTR) and will make it possible to determine the fundamental Virtual Behavioral Primitives (VBP) and specify the characteristics of the Virtual Environment (VE).

PHASE 4: Establishing the required VBP (Virtual Behavioral Primitives) and the EV characteristics.

As we have indicated, the Virtual Behavioral Primitives (VBP) are basic sensorimotor and cognitive activities that will enable the user to realize the functional $\mathrm{I}^{2}$ for which the system is designed. There are 4 main categories of VBP:

- Observe the virtual world;

- Move around in the virtual world;

- Interact in the virtual world;

- Communicate with others or with the application.

The useful Virtual Behavioral Primitives (VBP) are determined on the basis of the I2f indicated in the Functional Terms of Reference (FTR). For example, with regard to the virtual store for analyzing buying behavior [15], we determine three fundamental VBP: the observation of the product / three dimensional handling and the spatial orientation of the product / observation of the products on the shelf. We therefore also define two secondary VBP: the ease with which the customer moves and orients in the virtual store (VE) / the possibility of the customer picking up a product and then putting it back on the shelf or in the trolley. 


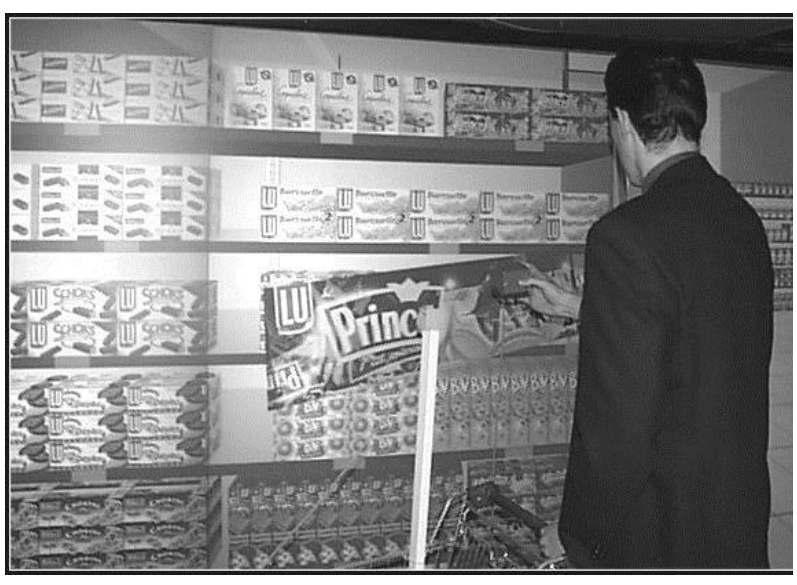

Figure 7. Virtual store for IN VIVO, designed and produced by the Ecole des Mines

ParisTech and by the company Sim Team (photo EMP)

Establishing the required Virtual Behavioral Primitives (VBP) has to be very precise: each VBP has to be analyzed to establish the minimum characteristics that will meet the I2f. For example, if an object has to be handled: should it be handled with two hands or one hand? With 6 degrees of freedom or less? With 360 degree rotation or less? Over what maximum translated distance? Should the weight and inertia be felt? Etc. However, determining the required VBP and their minimum characteristics should not in any way result in or presuppose technological solutions that will be determined in the next phase.

This phase should also allow the software characteristics to be established in order to create the virtual environment (VE) that meets the needs of the application and therefore the I2f. The VE should be mainly determined at the level of the entities making it up (objects, living beings, fluids, etc.) and at the level of the laws operating there physical laws (or not) (optical, mechanical, acoustic, etc.) - and the entities' behavioral laws (fixed or variable, deterministic or not, acoustic, etc.). This phase, for the software characteristics needed for the VE is in part related to determining the Virtual Behavioral Primitives (VBP), For example in a virtual store, if the user has to have a certain quality in visually observing the products (quantified by visual acuity), the display characteristics of the VE must be linked to the required visual quality.

The question of observation refers back to perception: "what information is needed and will be sufficient for the user to complete the task?" How does the user know that this or that action is required, how does the user know whether the action is successful or not? For example, a maintenance operator on a dangerous site must check that $\mathrm{s} / \mathrm{he}$ is in fact in nominal mode and that the procedure takes place as it should. S/he should be attentive to the signs that could change the situation into fail mode. It may be an element that is functioning abnormally (post condition not achieved), or an expected or unplanned sound, an abnormally high effort, etc. These questions establish which perceptive elements should be put in place and with what precision.

\section{DELIVERABLE 1: FTR-VR}

Phases 2, 3 and 4 result in writing an initial document, the Functional Terms of ReferenceVirtual Reality (FTR-VR). It is a "classic" FTR with in addition the definition of the Virtual Behavioral Primitives (VBP) and the Virtual Environment (VE) characteristics corresponding to the functional Sensorimotor Immersion \& Interactions (I2s).

PHASE 5 \& 6: Choice of the Cognitive Processes (CP) at the level of the I2c (Imported Behavioral Schema, metaphors, etc.) and Behavioral Interfaces (BI) at the level of the I2sm.

During this phase, the design team first works on the "cognitive" aspects of the system, while knowing that the exploitable Cognitive Processes (CP) for each Virtual Behavioral Primitives (VBP) are dependent on the Behavioral Interfaces (BI) that will be associated with the CP. For each VBP and on the level of the cognitive I2, either in principle or in relation to past experience in virtual reality, we look for the Imported Behavioral Schemas (IBS) that will provide efficient immersion and interaction. If it is impossible to find effective and technically achievable IBS at an affordable cost, we also have the choice of finding an appropriate metaphor, with or without sensorial or motor substitution, that is compatible with the functional $I^{2}$. In the example of the virtual store, for the three fundamental VBP, the decision is to make use of the visual observation schema in one fixed direction and the product grasping and spatial manipulation schema. For the two secondary VBP, the decision is to use the movement schema by pushing a trolley and the product grasping and spatial manipulation schema.

The design team has to also work on the sensorimotor $\mathrm{I}^{2}\left(\mathrm{I}^{2} \mathrm{sm}\right)$ on the basis of the desired Cognitive Processes (CP). The goal is to determine the senses and / or the motor responses as well as the artifact in the Behavioral Interface (BI), associated with Cognitive Processes chosen. It is recommended that the chosen artifacts (physical interfaces) are easily used by the target audience resulting in minimal learning time for users (for example no more than $10 \%$ of the time required to use the system). The team also determines what virtual environments will be used by the system. In the example of the virtual store, the following concepts are proposed: using a large projection 
screen in order to visualize the store on scale 1 ; using a 6 degree of freedom sensor in a cube for handling the products; using a real trolley to move around the store; realistic simulation of the store shelves and the high definition products so packaging information is readable, etc.

The choice of behavioral interfaces will be a compromise between the requirements of different factors, including:

The objectives of the application: in principle the way of reproducing - or not - a certain number of aspects in the task should not be decided on the basis of technological problems or attractions, but because they are considered useful in order to achieve the objectives of the desired application. Thus, the choice of instant movement of the "mouse click" type in the virtual place the user wants to go is both metaphorical and economic in terms of movement but does not allow the user to learn how to move or orientate him or herself. On the other hand, sometimes certain functionalities should not be reproduced in order to avoid overloading the user with information and allow him or her to concentrate on the task.

The technological and economic limitations: some interfaces cannot technically be produced or are too expensive in relation to the economic context in which we are working and given the uses and objectives of the application.

The usability and the cost related to learning how to use the interface: Virtual Reality interfaces attempt to allow habitual schemas to be used. Of course, the transposition leads to a loss of "naturalness". Sometimes, using a habitual schema needs a significant amount of time to learn; for example, the use of a treadmill in order to reproduce natural walking [20]. Nevertheless, the cost of learning to use the interface should be considered in relation to the objective sought. If these disadvantages are considered too great, it may be helpful to take a more metaphorical approach.

User experience and profile in other areas: depending on the audience and its experience, some interfaces may be more easily used than others. For example, if the users play video games, a Wiimote could easily be integrated into the system with a low user learning "cost".

Compatibility between the interfaces: if each behavior primitive is taken separately, it is possible to give each one the optimal interface, but there is then a risk of difficulties in compatibility arising. For example, using a treadmill may be incompatible with wearing a head mounted display and difficult in a cave with six sides.

\section{DELIVERABLES 2 \& 3: Cognitive Terms of Reference (CTR) \& Concepts (Design file)}

Phase 5 results in writing the Cognitive Terms of Reference (CTR) which complements the Functional Terms of Reference (FTR) with a presentation of the cognitive $\mathrm{I} 2\left(\mathrm{I}^{2} \mathrm{c}\right)$ and the Imported Behavioral Schema (IBS) used by the system. During the creative phase, the design team defines all the concepts that seem relevant in producing the system. The choice of concepts that will be selected and implemented are often the strategic responsibility of the project's sponsor. The main principle of the design file is therefore presenting an overall summary of all the envisaged concepts, with an evaluation of their advantages and disadvantages, in order to allow the decision makers to make their choice.

PHASE 7: Definition of the BI (Behavioral Interfaces), VE (Virtual Environment) and BSA (Behavioral Software Assistance)

Once the concepts have been defined, the design team now has to exactly define the architecture and the expected characteristics of the future system in order to allow the programmers to produce a prototype.

The metrological characteristics of the physical interfaces have to be defined to be compatible with psychophysical characteristics of the senses and the motor responses, in relation to the desired I2 sensorimotor. The team may decide to use standard interfaces or develop specific interfaces for the system. The technological and competitive intelligence put in place at the start of the project is very useful during this definition phase.

As applicable, Behavioral Software Assistance (BSA) is designed to improve the cognitive $\mathrm{I} 2\left(\mathrm{I}^{2} \mathrm{c}\right)$ (remember that there are still sensorimotor inconsistencies and that the BSA may partially allow the subject's brain to accept these inconsistencies).

DELIVERABLE 4: VE, BI and BSA (Virtual Environment, Behavioral Interfaces and Behavioural Software Assistance)

The definition file brings together details of all the elements in the system. It is aimed at the people that will finalize the first system prototype, first and foremost the computer specialists. If artifacts are created (physical interfaces), the computer specialists will be assisted by electronics engineers, mechanics, roboticists, members of the design team or act as subcontractors. System plans, mock ups or descriptive schemas complete the definition file. 


\section{PHASE 8: Programming (Prototyping)}

A system incorporating Virtual Reality (VR) relies first and foremost on a computer environment (computer, work station, cluster of computers, etc.) to which all the peripheral devices needed for the system are connected. The prototype phase is therefore principally the responsibility of the computer specialists. Even if some software presents an interface that is easily accessible to non programmers, it is illusory to think that a team without a computer specialist would be able to develop an efficient system. With ongoing contact with the computer specialists, the project leader ensures the system definition file is followed and will, if needed, learn to communicate with them (computer specialists sometimes have a vision of the system that is structured around blocks of lines of codes and think more in terms of producing computer functions rather the global function of the system) [21]. Regular validation stages must be planned during this phase in order to match the design team's requirements with the computerization possibilities (which are sometimes overestimated).

\section{DELIVERABLE 5: System prototype}

The prototype should benefit from all the functionalities expected from the system. As in the software industry, each prototype is given a version number (we start with "beta" versions and then go on to versions $1.0,1.1$, etc.). In fact, as it is simple to change the program code, the prototypes are not "stable" and very quickly evolve (as soon as the first inevitable bugs have been corrected). During the first prototype trials exchanging files by Internet allows the computer specialists to very quickly respond to the design team's requests and is one of the advantages of working virtually.

\section{PHASE 9: Evaluation}

It is essential to evaluate a system incorporating Virtual Reality and it must be done rigorously. It should be done on three levels, testing in the following order and without missing a level: the sensorimotor I2, the cognitive I 2 and the functional $\mathrm{I} 2$

For the sensorimotor $\mathrm{I} 2\left(\mathrm{I}^{2} \mathrm{sm}\right)$, the "metrological" characteristics of the artifacts used are measured (physical interfaces). We then compare them to average human characteristics in order to determine the relative sensorimotor deficit for each sense and for each motor response. We then verify that this deficit is compatible with the desired cognitive $\mathbf{I}^{2}$. The most important metrological characteristics for the interfaces are: precision, sensitivity, the scope of use, the frequency of measurement and response time (do not rely on the characteristics given by the interface constructor as these depend on the conditions of use).

For cognitive $\mathrm{I} 2\left(\mathrm{I}^{2} \mathrm{c}\right)$, the evaluation is more difficult to quantify. The time taken to learn how to use the behavioral interfaces is a good indicator. If the user masters the system very rapidly, it means that the chosen CP (Cognitive Process) is effective. Classic ergonomic tests are recommended and preferably should be entrusted to ergonomists.

For functional I2 (I2f) we refer to the Virtual Behavioral Primitives (VBP) defined in the Functional Terms of Reference (FTR-VR). When possible, the evaluated function in the system should be compared with the similar function in the real world. In the example of the virtual store: does the consumer behave similarly in the virtual store and a real store?

Specialists from several disciplines and a representative sample of users (user-designer and end user) will be called on to conclude this phase.

\section{PHASE 10: Release \& Exploitation}

The evaluation phase (Phase 8) loops into the various phases in the method if there is a deficiency in the system in relation to certain expected functions. The present exploitation phase only begins once the system has been "approved" by the sponsor. As it is relatively easy to modify a computer program, systems incorporating Virtual Reality often benefit from frequent updates and continual development through the lifetime of the system.

\section{PHASE 11: Improvements (Phase 7 loop)}

Day to day operation of the system generally results in users wanting additional functions or new virtual environments (presentation of the product in different locations) above all when designing innovative products. The additional expected functionalities then have to be clearly defined, the costs and the development lead in time evaluated before beginning a new programming phase. A further evaluation phase is then recommended, but is not obligatory if the new functionalities do not fundamentally change the way the system is used. 


\section{CONCLUSION}

We are proposing a new method for designing systems using virtual reality (VR) technologies. The result of the experience gained by various research teams, this design method benefits from contributions from current VR research. Our objective is to validate and continuously move forward such multidisciplinary design team methods [22].

Our next goal will be to adapt this method to a cocreation context when final users are involved in the design team to create a new compelling Virtual Reality experience. Through the study of the User Experience within an immersive Virtual Reality system (such as a Cave $\AA$ for example), we also propose to address the challenge of augmenting the user experience. In order to do this, we first need to determine all the factors that may influence (positively or negatively) the user experience. These factors can be related to VR systems (hardware), 3D content (software) or can be related to the use of $3 \mathrm{D}$ content within the VR system. Understanding these factors will help us to develop a updated design methodology of VR systems (from both technical and 3D content points of view) that will, by taking into account these factors, augment the user experience

The success of the $\mathrm{I}^{2} \mathrm{I}$ proposed method is based to a large extent on the efficiency of the project leader who heads the design team. Convinced of the performance of the $I^{2} I$ method, the project leader has to identify any obstacles, define the team's and users' needs, motivate the participants... Assisted by computers and networks, collaborative work in a multidisciplinary team should be encouraged. Even in the virtual field, people are central to the process.

\section{REFERENCES}

[1] S. Richir, P. Fuchs, D. Lourdeaux, C. Buche, R. Querrec, (2013), An industrial approach to design compelling VR and AR experience, Conference 8649 The Engineering Reality of Virtual Reality, IS\&T/SPIE Electronic Imagine Conference, 3 - 7 February 2013, Hyatt Regency San Francisco Airport Hotel, Burlingame, California United States, Proceedings of SPIE Volume 8649.

[2] Richir Simon, (2003). Les technologies de la réalité virtuelle pour la conception de produits, Mémoire d'Habilitation à Diriger des Recherches, Université d'Angers.

[3] Brunnett G., Coquillart S. and Welch G. (2008). 08231 Abstracts Collection, Virtual Realities, Dagstuhl Seminar, http://drops.dagstuhl.de/opus/volltexte/200 8/1634/

[4] Lindeman, R.W., Beckhaus, S., (2009) Crafting Memorable VR Experiences using Experiential Fidelity XXXX

[5] Beckhaus, S., Lindeman, R.W. (2010) Experiential Fidelity: Leveraging the Mind to Improve the VR Experience In Book: "Virtual Realities". Dagstuhl Seminar 2008. Springer.

[6] Stuart R., (2001). The Design of Virtual Environments », Barricade Books Inc., ISBN 1-56980-207-6, 2001.

[7] W.R. Sherman, A.B. Craig, (2003). Understanding Virtual Reality, Elsevier Science USA, ISBN 1-55860-353-0.

[8] Chen J., Bowman D.A., (2009). Domainspecific design of $3 \mathrm{~d}$ interaction techniques: An approach for designing useful virtual environment applications. Presence: Teleoperators and Virtual Environments. Volume 18, Issue 5, pp. 370-386.

[9] Fuchs P. et al. (2003). Le Traité de la réalité virtuelle, version 2 , éditeur « Les presses de l'Ecole des Mines de Paris », Paris.

[10] Beckhaus, S., Lindeman, R.W. (2010) Experiential Fidelity: Leveraging the Mind to Improve the VR Experience In Book: "Virtual Realities". 2011, pp 39-49

[11] Rabardel P., (1995). Les hommes et les technologies, une approche cognitive des instruments contemporains, Paris, Colin.

[12] Maman D. (1998). Recalage de modèles tridimensionnels sur des images réelles. Thèse de doctorat de l'Ecole des Mines de Paris, 1998.

[13] Piaget J., Chomsky N., (1979). Théories du langage, théories de l'apprentissage, Seuil, 1979.

[14] Fuchs P. (2000). Approche théorique de la réalité virtuelle et son exploitation, Mémoire d'Habilitation à Diriger des Recherches, Ecole des Mines, Paris.

[15]Fuchs P., Nashahibi F., Lourdeaux D. (1999). A theoretical approach of the design and evaluation of a virtual reality device, VRIC Laval Virtual proceedings, 3-4 Juin 1999, Laval, pp. 11-20.

[16] Corsi P., Christofol H., Richir S., Samier H. (2006). Innovation Engineering - the power of intangible networks, ISTE 2006, 400 p., ISBN 13 : 978-1-905209-55-2.

[17]Richir Simon, (1994). La conception de produits nouveaux dans l'industrie du jouet, 174 p., Thèse de Doctorat, Génie Industriel, Ensam, 1994, n94-13.

[18]Fuchs P., Moreau G., Papin J.P. (2001). Le Traité de la réalité virtuelle, 540 p., 
www.ensmp.fr/Fr/Services/PressesENSM $\mathrm{P}$, editor « Les presses de l'Ecole des Mines de Paris », ISBN 2-911762-34-7, Paris.

[19] Yannou Bernard, Christofol Hervé, Jolly Daniel, Troussier Nadège, (2008). La conception industrielle de produits, Vol.3 - Ingénierie de l'évaluation et de la décision, série Productique, Hermes Sciences Lavoisier, 321p., ISBN 978-27462-1920-4.

[20] Lourdeaux D., (2001), Réalité Virtuelle et formation : conception d'environnements virtuels pédagogiques. Thèse de doctorat en Informatique temps réel, Robotique et Automatique, École des Mines de Paris.

[21] Richir S., Fuchs P., (2006a). La méthode I2I, Interaction et Immersion pour l'Innovation. Méthode de conception pour les applications exploitant les environnements virtuels. Editions Techniques de l'ingénieur, Rubrique Réalité Virtuelle, Paris, 20 p.

[22] Richir S., Fuchs P., (2006b). Réalité Virtuelle et Conception, Chapitre du volume 4, les applications de la réalité virtuelle, du traité de la réalité virtuelle, Fuchs P., MOREAU G. et 72 auteurs, 4 volumes, Les Presses de l'Ecole des Mines de

Paris, <http://www.esnmp.fr/Presses>www.esnm p.fr/Presses, mars 2006, ISBN 2-91176265-7.

\section{GLOSSARY}

$\mathbf{B I}=$ Behavioral Interface $(\mathrm{IC}) ; \mathbf{B S A}=$ Behavioral Software Assistance (ALC) ; IBS = Imported Behavioral Schema (SCI) ; FTR = Functional Terms of Reference (CDCF) ; BI = Behavioral Interfaces (IC) ; $\mathbf{V E}=$ Virtual Environment $; \mathbf{V B P}=$ Virtual Behavioral Primitives (PCV); CP $=$ Cognitive Processes (PC); CTR = Cognitive Terms of Reference ; UPI = User Perceptual Interface; $\mathbf{I}^{2} \mathbf{f}=$ Functional Immersion \& Interaction ; $\mathbf{I}^{2} \mathbf{c}=$ Cognitive Immersion \& Interaction ; I'f $=$ Sensorimotor Immersion \& Interaction. 